\title{
Étudier Claude Simon au lycée
}

Studying Claude Simon in Secondary School

\section{Cécile Yapaudjian-Labat}

\section{(2) OpenEdition}

Journals

Édition électronique

URL : http://journals.openedition.org/ccs/1972

ISSN : 2558-782X

Éditeur :

Presses universitaires de Rennes, Association des lecteurs de Claude Simon

Édition imprimée

Date de publication : 30 septembre 2019

Pagination : $57-71$

ISBN : 978-2-7535-7795-4

ISSN : $1774-9425$

\section{Référence électronique}

Cécile Yapaudjian-Labat, «Étudier Claude Simon au lycée », Cahiers Claude Simon [En ligne], 14 | 2019, mis en ligne le 30 septembre 2020, consulté le 10 octobre 2020. URL : http://journals.openedition.org/ ccs/1972 


\title{
ÉTUDIER CLAUDE SIMON AU LYCÉE
}

\author{
Cécile Yapaudjian-Labat \\ Aix-Marseille université
}

On le sait, beaucoup diront que lire Claude Simon n'est pas une mince affaire ${ }^{1}$. Dans cette entreprise, la participation active du lecteur, $s^{\prime}$ il ne veut pas rester extérieur au puissant charme du texte simonien, est requise. Dans ces conditions, et alors que les instructions officielles du premier comme du second degré insistent sur la nécessité de susciter chez les élèves le plaisir de lire, faire étudier Simon est-il envisageable? Judicieux? Mais aussi : quelle est aujourd'hui la visibilité de Simon dans le secondaire? Pour répondre à ces interrogations, trois pistes au moins méritent d'être explorées. En limitant l'investigation au niveau du lycée, il s'agira de se demander quelle est la place réservée à Simon, d'abord dans les manuels scolaires, ensuite dans la pratique de classe proprement dite. Une entrée dans l'œuvre par le biais des ateliers d'écriture créative sera enfin proposée.

\section{UNE PRÉSENCE EN DEMI-TEINTE DANS LES MANUELS SCOLAIRES}

Quasiment absente jusque-là des manuels de lycée, l'œuvre de Claude Simon, sous la forme d'extraits², y trouve sa place à partir du début des années 2000. La cause la plus directe de ce tout nouvel intérêt est à rechercher sans doute

1. Nous nous permettons de renvoyer sur ce sujet au numéro que nous avons coordonné : Tangence-Autour de Claude Simon : trajets de lectures, université du Québec à Rimouski et université du Québec à Trois-Rivières, $\mathrm{n}^{\circ} 112,2016$.

2. L'anthologie Lagarde et Michard propose un extrait de La Route des Flandres dans sa réédition augmentée de 1988, peu après le Nobel, donc. Précurseur, le manuel Littératures dirigé par M.-H. Prat et M. Aviérinos et publié chez Bordas en 1997 propose un extrait des Géorgiques dans son second tome, consacré aux XIX ${ }^{e}$ et $\mathrm{Xx}^{\mathrm{e}}$ siècles. 
dans le choix d'inscrire le roman de 1960, La Route des Flandres, au programme de l'agrégation de lettres en 1998 (conjointement au concours d'entrée à l'ENS). Adoubée par l'institution, l'œuvre devient alors légitime et de ce fait susceptible d'être étudiée par les lycéens français.

Nous avons consulté la totalité des manuels de français de classes de Seconde et Première dits "livre unique ", c'est-à-dire abordant les questions de langue, l'analyse littéraire des textes, l'histoire littéraire et dans une moindre mesure l'histoire de l'art, parus entre 2001 et 2016, présents dans les bibliothèques de deux écoles supérieures du professorat et de l'éducation, celle de Paris et celle d'Aix-en-Provence. Les constats sont les suivants : sur les quatre grandes vagues de publication de manuels de français de lycée que sont les années 2001, 2007, 2011 et 2015, seuls les ouvrages parus lors des deux premières, en 2001 et 2007, ont réservé une place à des extraits de romans de Simon : deux manuels en 2001, un en 2004, cinq en 2007. Les plus gros éditeurs sont concernés : Nathan, Hachette, Hatier, Bordas, Delagrave et Bertrand-Lacoste. Le niveau dominant est celui de Première.

Les extraits choisis ont permis aux concepteurs de manuels de proposer de multiples voies d'accès au texte. Un rapide parcours des extraits retenus ainsi que de l'appareil didactique accompagnant ces derniers permet de le constater dans le tableau ci-après. 


\begin{tabular}{|c|c|c|}
\hline Manuel & Texte de Simon choisi & Entrée didactique \\
\hline $\begin{array}{l}\text { D. Stissi (dir.), } \\
\text { Français Première, } \\
\text { Delagrave, } 2001 .\end{array}$ & $\begin{array}{l}\text { Histoire, incipit (Hist., } \\
\text { p. 147-148). }\end{array}$ & «Romans à la première personne " (p. 292). \\
\hline $\begin{array}{l}\text { C. Eterstein (dir.), } \\
\text { Les Nouvelles } \\
\text { pratiques du } \\
\text { Français - } \\
\text { Première, } \\
\text { Hatier, } 2001 .\end{array}$ & $\begin{array}{l}\text { Leçon de choses, } \\
\text { fin de la section } \\
\text { "Expansion " (des- } \\
\text { cription d'un tableau } \\
\text { de Monet reproduit } \\
\text { sur un calendrier) } \\
(L C, \text { p. } 581) .\end{array}$ & $\begin{array}{l}\text { «Les réécritures ». La description : hypotypose } \\
\text { et tableau. }\end{array}$ \\
\hline $\begin{array}{l}\text { J. Jordy (dir.), } \\
\text { Français } \\
\text { Première, } \\
\text { Bertrand- } \\
\text { Lacoste, } 2007 .\end{array}$ & $\begin{array}{l}\text { - Histoire, excipit } \\
\text { (Hist., p. 415-416). } \\
\text { - Extrait du Discours } \\
\text { de Stockholm (DS, } \\
\text { p. 894-895) } \\
\text { - Extrait de la préface } \\
\text { à Orion aveugle (OA, } \\
\text { p. 1181-1183). }\end{array}$ & $\begin{array}{l}\text { - Littérature et histoire (p. 128-129). } \\
\text { - Repères : "Personnage - Histoire - Fiction" } \\
\text { (p. 134-135) : le réalisme. }\end{array}$ \\
\hline $\begin{array}{l}\text { D. Rincé (dir.), } \\
\text { Français Première, } \\
\text { Nathan, } 2007 .\end{array}$ & $\begin{array}{l}\text { La Route des Flandres, } \\
\text { première description } \\
\text { du cheval mort (RF, } \\
\text { p. 210-211). }\end{array}$ & $\begin{array}{l}\text { "Le roman éclaté » (p. 330) } \\
\text { - À partir de quel point de vue s'effectue la } \\
\text { vision dans ce passage? Précisez la position du } \\
\text { personnage. Quelles en sont les conséquences sur } \\
\text { le mode de perception? } \\
\text { - Comment l'écriture marque-t-elle la fragmen- } \\
\text { tation et la fluctuation de la vision? Observez } \\
\text { notamment le jeu étrange des parenthèses. } \\
\text { - En quoi les choses vues accentuent-elles cette } \\
\text { impression de fragmentation? Reliez votre } \\
\text { réponse au contexte historique de la scène. } \\
\text { - Dans quelle mesure cette écriture vous semble-t- } \\
\text { elle également proche du cinéma? De quels mou- } \\
\text { vements de caméra pourrait-on alors rapprocher } \\
\text { le jeu de regard du personnage et l'unique longue } \\
\text { phrase qui constitue l'extrait? } \\
\text { - Comment s'effectue, dans l'ensemble du pas- } \\
\text { sage, le rapprochement, jusqu'à la confusion, } \\
\text { entre hommes et animaux, entre êtres animés et } \\
\text { choses? Expliquez comment le tout est marqué } \\
\text { par la présence envahissante de la mort. } \\
\text { - De quelle conception moderne de la réalité, et } \\
\text { de la perception que l'on peut en avoir, cet extrait } \\
\text { de roman témoigne-t-il? } \\
\text { - Bio-bibliographie de Simon (p. 562). }\end{array}$ \\
\hline
\end{tabular}




\begin{tabular}{|c|c|c|}
\hline $\begin{array}{l}\text { C. Eterstein (dir.), } \\
\text { Les Pratiques } \\
\text { du Français- } \\
\text { Première, Hatier, } \\
2007 .\end{array}$ & $\begin{array}{l}\text { La Route des Flandres, fuite } \\
\text { du soldat après l'anéantisse- } \\
\text { ment de son escadron }(R F \text {, } \\
\text { p. 302-303). }\end{array}$ & $\begin{array}{l}\text { La construction littéraire du personnage } \\
\text { dans le cadre d'une étude de corpus sur le } \\
\text { thème du héros à la guerre : Stendhal, La } \\
\text { Chartreuse de Parme; Hugo, Les Misérables; } \\
\text { Céline, Voyage au bout de la nuit. } \\
\text { Questions sur le texte de Simon: } \\
\text { - À quoi se réduit la connaissance des per- } \\
\text { sonnages? } \\
\text { - Quelle est l'influence du cinéma sur la } \\
\text { description littéraire? }\end{array}$ \\
\hline $\begin{array}{l}\text { L. Carpentier } \\
\text { (dir.), Soleils } \\
\text { d'encre - Lettres } \\
\text { et langue - } \\
\text { Première, } \\
\text { Hachette éduca- } \\
\text { tion, } 2007 .\end{array}$ & $\begin{array}{l}\text { Leçon de choses, } \\
\text { "Générique " ( } L C, \text { p. } 557- \\
558) \text {. }\end{array}$ & $\begin{array}{l}\text { Étude des mécanismes de la description, } \\
\text { réflexion sur les parenthèses et sur le sens } \\
\text { du « etc. ". }\end{array}$ \\
\hline $\begin{array}{l}\text { J.-P. Aubrit et } \\
\text { D. Labouret } \\
\text { (dir.), Textes et } \\
\text { Perspectives - } \\
\text { Français/ } \\
\text { Littérature } \\
\text { Seconde, Bordas, } \\
2004 .\end{array}$ & $\begin{array}{l}\text { L'Acacia, ch. viII (1939- } \\
\text { 1940), "Le cadavre noir de } \\
\text { l'Histoire " ( } A, \text { p. 1159- } \\
\text { 1160). }\end{array}$ & $\begin{array}{l}\text { - Comment le récit rend-il sensible l'an- } \\
\text { goisse des personnages? } \\
\text { - Quels sont les effets produits par l'inter- } \\
\text { ruption des parenthèses? } \\
\text { - Comment le texte conduit-il progressive- } \\
\text { ment vers une lecture allégorique de la scène? } \\
\text { - À quoi l'Histoire est-elle assimilée? } \\
\text { - D'où provient la violence de la dernière } \\
\text { phrase? Identifiez les procédés d'écriture } \\
\text { qui y contribuent. }\end{array}$ \\
\hline $\begin{array}{l}\text { C. Desaintghis- } \\
\text { lain, } \\
\text { C. Morisset, } \\
\text { P. Wald Lasowski, } \\
\text { Littérature- } \\
\text { Français, Nathan, } \\
2007 .\end{array}$ & $\begin{array}{l}\text { La Route des Flandres, le } \\
\text { narrateur regarde autour } \\
\text { de lui après la fuite lors de } \\
\text { l'anéantissement de son es- } \\
\text { cadron }(R F, \text { p. 305-306). }\end{array}$ & $\begin{array}{l}\text { Point bio-bibliographique. "La dilatation } \\
\text { de l'univers romanesque". } \\
\text { Questions sur l'extrait : (groupement de } \\
\text { textes autour du Nouveau Roman) : } \\
\text { - Repérez les indicateurs spatio-temporels } \\
\text { dans le récit. Quelles sont les étapes qui le } \\
\text { constituent? } \\
\text { - Analysez la présence des sens dans le } \\
\text { texte. Quels sens la perception du narrateur } \\
\text { privilégie-t-elle? } \\
\text { - Relevez et étudiez le champ lexical de la } \\
\text { petitesse dans le texte. A quoi correspond } \\
\text { cette présence? } \\
\text { - Quel est l'événement qui vient inter- } \\
\text { rompre la rêverie du narrateur? À quel } \\
\text { contexte renvoie-t-il? } \\
\text { - En quoi cet extrait constitue-t-il un } \\
\text { monologue intérieur? }\end{array}$ \\
\hline
\end{tabular}


Relevons d'abord que seuls quatre romans de Simon sont retenus : La Route des Flandres (1960), Histoire (1967), Leçon de choses (1975) et L'Acacia (1989). Si le choix des deux premiers se justifie au moins par la reconnaissance publique et critique qu'ils ont obtenue - prix de L'Express pour le premier, prix Médicis pour le second -, la présence d'extraits de Leçon de choses peut sembler plus surprenante dans la mesure où ce roman appartient à la période la plus formaliste de Simon, celle qui est considérée comme la moins accessible. Or, c'est précisément pour leur intérêt d'un point de vue formel que ces extraits ont été retenus. Dans ce roman, la description est première au sens où c'est elle qui systématiquement génère la fiction : l'étude de la description comme tableau, de l'hypotypose, prend tout son sens ici. En outre, l'auteur fait de sa description un ample mouvement sans fin. L'ouverture du roman consiste d'ailleurs en une longue phrase descriptive potentiellement non close puisqu' elle a pour dernier mot " etc. ". Il s'agit ainsi de dire tous les possibles du réel, de montrer le plus possible le monde, dans tous ses détails, dans toutes les directions - par l'usage récurrent des parenthèses notamment. Or, l'analyse de ces procédés permet de faire prendre conscience au jeune lecteur du travail spécifique de l'écriture, de mettre en correspondance très concrètement l'intention de l'écrivain, les moyens mis en œuvre et les effets produits. La question posée dans plusieurs ouvrages de méthode sur les effets produits par l'absence de ponctuation va dans le même sens.

À partir des deux extraits de La Route des Flandres, c'est le regard singulier du narrateur simonien qui, cette fois, est mis en relief : une "vision fragmentaire ", celle d'une conscience qui ne peut qu'enregistrer le " chaos du monde ", le réel qui l'entoure, comme le ferait une simple caméra (" quelle est l'influence du cinéma?"), sans accéder au sens, à la compréhension de ce monde. L'étude du point de vue, dans les livres de Simon et dans La Route des Flandres en particulier, permet de dessiner les contours d'un personnage romanesque problématique. Certes, ce personnage emprunte beaucoup de ses caractéristiques au héros épique puisqu'il s'agit d'un jeune homme qui part à la guerre et se retrouve dans un régiment de cavalerie. Mais si la mémoire de l'épopée est bien présente dans le roman, c'est pourtant la dégradation des valeurs épiques et de l'héroïsme qui se trouve explicitement affirmée. Il est intéressant alors d'étudier un corpus associant le passage de La Route des Flandres où le personnage, après l'embuscade de son escadron, fuit à quatre pattes dans les fourrés, comme sans conscience, exclusivement sensible à ce qu'il perçoit (le sol, la végétation, le chant d'un oiseau) à des extraits de scènes guerrières 
de La Chartreuse de Parme - le fameux passage dans lequel l'enthousiaste Fabrice, à Waterloo, est perçu par un narrateur ironique mais bienveillant -, des Misérables - les Thénardier détrousseurs de cadavres sur le champ de bataille - et du Voyage au bout de la nuit - un soldat fait l'expérience de l'horreur de la Première Guerre. Ce groupement de textes permet aux élèves de comprendre la relation étroite qui existe entre la remise en cause du récit épique et avec lui du héros traditionnel, et l'Histoire - l'histoire du $\mathrm{Xx}^{\mathrm{e}}$ siècle surtout, jalonnée par des situations de guerre et de destruction de l'homme inédites. Cette Histoire a rendu le récit épique indécent, voire impossible. Le roman de Claude Simon, exemplairement, témoigne de ce bouleversement et conduit les élèves, à partir de l'étude du personnage et de sa vision, à une réflexion sur les relations entre "littérature et histoire ». C'est dans une perspective proche qu'est étudié l'extrait de L'Acacia dans lequel l'Histoire, perçue d'abord métaphoriquement comme un cadavre noir, devient progressivement une «vieille ogresse " digérant les soldats puis les rejetant par son " anus ridé [...] sous forme d'excréments» (p. 1159-1160).

Susciter cette réflexion apparait d'ailleurs comme l'objectif premier dans le manuel de 2007 dirigé par Jean Jordy, cette fois à partir de l'excipit d'Histoire, mais aussi de deux extraits de Simon sur sa pratique de l'écriture et sur la littérature : le Discours de Stockholm et la Préface manuscrite d'Orion aveugle. Dans ces deux derniers textes, Simon s'applique entre autres à présenter ce qu'est pour lui le réalisme. C'est dans la perspective de retracer l'évolution du personnage dans le temps que le manuel propose une fiche "Repères " s'intéressant aux relations entre " Personnage - Histoire - Fiction » à travers la question du " réalisme » en littérature.

Deux dernières remarques : seuls les deux ouvrages parus chez Nathan en 2007 consacrent une notice bio-bibliographique à Simon, avant l'extrait étudié ou dans l'index des auteurs. Malgré le Nobel, malgré la reconnaissance internationale, malgré l'entrée de son œuvre dans la prestigieuse collection de la Pléiade en 2006, celui qui est considéré aujourd'hui comme l'un des plus grands romanciers du $\mathrm{Xx}^{\mathrm{e}}$ siècle est certes présent dans quelques ouvrages de lycée mais n'a pas la place ni le rayonnement auxquels il pourrait prétendre. Or, il semble que la situation régresse brutalement après 2007 : les extraits de Simon disparaissent alors purement et simplement des nouvelles éditions de manuels. Il est peu probable que cette disparition ait à voir avec l'arrivée des nouveaux programmes de français au lycée en 2010. Ces derniers consacrent en effet un objet d'étude, pour la classe de Première, au « Personnage de ro- 
man du XVII ${ }^{\mathrm{e}}$ siècle à nos jours ", dans lequel les textes de Simon ont bien leur place. S'il est donc difficile d'interpréter cette suppression, il est à signaler que les auteurs contemporains désormais convoqués autour des questions du réalisme, des relations entre fiction et Histoire, sont pour la plupart des auteurs qui ont revendiqué leur attachement à l'œuvre de Claude Simon : François Bon et Sylvie Germain ${ }^{3}$ notamment.

\section{UN AUTEUR PEU VISIBLE DANS LES CLASSES}

Cette présence limitée dans les livres scolaires se transforme en véritable absence dans la réalité des classes de lycée. La raison essentielle est peut-être à rechercher dans la méconnaissance des enseignants eux-mêmes de l'œuvre de Simon malgré l'intérêt qu'elle suscite à l'université, ou de leur frilosité à faire étudier à leurs élèves une prose réputée difficile.

Depuis une vingtaine d'années maintenant, pourtant, les romans de Claude Simon font l'objet d'un enseignement régulier dans les universités françaises. Une enquête menée auprès de quatorze enseignants-chercheurs et enseignants en classes préparatoires aux grandes écoles et spécialistes de l'œuvre de Claude Simon ${ }^{4}$ permet de mettre en évidence un certain nombre d'éléments. Les romans le plus fréquemment étudiés ${ }^{5}$ sont d'abord La Route des Flandres, travaillée par dix enseignants différents et qui plus est au moins à deux reprises (dont l'année 1998 où le roman était au programme de l'agrégation), Le Vent et L'Acacia (6 enseignants) et Le Tramway (4). Viennent ensuite L'Herbe, Le Palace, Histoire et Les Géorgiques (2) et enfin Le Jardin des Plantes, Archipel et Nord, La Bataille de Pharsale, Les Corps conducteurs, L'Invitation et Triptyque (1). En outre, des extraits, essentiellement de La Route des Flandres, Le Vent, L'Herbe, Les Géorgiques, et L'Acacia sont régulièrement étudiés. Cet enseignement concerne tous les niveaux, de la première année de licence au master 2, mais il est prédominant en licence 3 et en master recherche. Dernier point : les universités dans lesquelles ces cours se sont déroulés sont nombreuses et harmonieusement réparties sur le territoire fran-

3. Dans O. Marais (dir.), Français I 1e - L'écho des Lettres, Belin, 2016.

4. Spécialistes en ce sens qu'ils ont tous consacré un ouvrage publié et/ou une thèse en tout ou partie à l'œuvre de Simon et qu'ils ont écrit de nombreux articles sur le romancier. Parmi eux, dix enseignent à l'université depuis 20 ans ou plus, quatre depuis au moins 10 ans.

5. Les enseignants-chercheurs et enseignants de CPGE ont répondu aux questions suivantes, par mail : Avez-vous déjà consacré l'un de vos cours à un roman de Claude Simon? Si oui, le(s)quel(s) ? Pour quel niveau d'enseignement? Dans quelle université? En quelle(s) année(s)? 
çais : Nice, Aix, Avignon, Perpignan, Toulouse, Le Havre, Paris 3, Paris 4, Paris 7, Paris 10, Lille, Grenoble. Tenons compte aussi du fait que tous les enseignants-chercheurs contactés n'ont pas répondu à l'enquête.

En outre, du côté des concepteurs des programmes de l'agrégation de Lettres modernes, l'intérêt pour l'auteur ne décroît pas depuis 1998 : vingt ans après La Route des Flandres ${ }^{6}$, un second roman de Simon, L'Acacia, est à nouveau inscrit, cette fois au programme de littérature comparée ${ }^{7}$ des sessions 2018 et 2019. Cela signifie que Simon est considéré comme un écrivain incontournable devant être bien connu des futurs enseignants.

Mais est-ce bien le cas? Sur deux groupes de 38 et 27 étudiants de master 1 MEEF (Métiers de l'enseignement, de l'éducation et de la formation) préparant le CAPES de lettres modernes ${ }^{8}$, tous ont déclaré connaître le nom de Claude Simon. Mais seulement un étudiant passé par une classe préparatoire littéraire a reconnu avoir lu " en entier " un ouvrage de Simon étudié en khâgne, La Route des Flandres, mais sans plaisir et quasiment "sous la contrainte ». Il faut ajouter à cet état des lieux la réputation d'auteur difficile qui poursuit Simon. Rappelons seulement sur ce dernier point les réactions de la presse française au moment de l'attribution du prix Nobel en 1985 : certains critiques littéraires présentent l'œuvre, alors encore méconnue du grand public, comme étant d'un "accès peu facile " ou encore comme "n' [étant] pas à la portée de tous ceux qui peuvent lire ${ }^{9}$ ". Simon fait d'ailleurs allusion à ces commentaires dans son Discours de Stockholm (p. 889).

La connaissance ou l'intérêt insuffisants des futurs enseignants et les déclarations liées à la difficulté de lecture de l'œuvre ne contribuent pas à favoriser l'étude de textes de Claude Simon au lycée. Le constat dans les classes, justement, est sans appel. Romans comme extraits de Simon semblent n'être quasiment jamais étudiés. Dans tous les cas, depuis 2000, aucun corpus de textes proposé à l'écrit de l'épreuve anticipée de français, toutes séries confondues, n'a comporté un extrait tiré d'un roman de Simon. Par ailleurs, interrogés sur la présence ou l'absence de textes de Claude Simon dans les listes de baccalauréat des candidats (toutes séries confondues là encore), dix-neuf enseignants

6. Roman également au programme du concours de l'École normale supérieure de Lyon pour les spécialistes en Lettres modernes en 2017.

7. Ajoutons encore qu'un extrait de L'Acacia figurait dans le corpus constitutif de l'épreuve de didactique lors de la session 2013 de l'agrégation interne de Lettres modernes.

8. ESPE de Paris/université Paris-Sorbonne, année universitaire 2015-2016 et ESPE Aix-Marseille, site d'Aix/AMU université, année universitaire 2016-2017.

9. Resp. La Vie, 24 octobre 1985, et France Soir, 18 octobre 1985. 
de lycée membres de jurys à l'oral des épreuves anticipées de français ont répondu négativement ou ne se sont pas souvenus d'avoir déjà rencontré un texte de cet auteur dans une liste, a fortiori d'avoir interrogé un candidat sur un extrait de Simon ${ }^{10}$.

Si quelques initiatives sont à signaler de la part d'enseignants de lettres de lycée et même de collège ${ }^{11}$, elles restent cependant marginales. Ce constat nous a conduite à nous demander comment susciter chez les enseignants en formation initiale le désir de faire étudier les textes de Simon à leurs élèves. Notre pratique de l'animation d'ateliers d'écriture auprès d'étudiants et étudiants stagiaires de lettres nous a incitée à proposer d'entrer dans ces textes par l'écriture créative.

\section{UNE PROPOSITION D'ENTRÉE DANS L'CEUVRE : L'ATELIER D'ÉCRITURE CRÉATIVE ${ }^{12}$}

Si l'atelier d'écriture nous semble constituer une voie d'accès à privilégier pour conduire des lycéens mais aussi des collégiens à lire Claude Simon ${ }^{13}$,

10. Dix-neuf enseignants de lettres modernes en classes de lycée interrogés par mail, répartis sur cinq académies différentes : Toulouse, Montpellier, Paris, Orléans-Tours, Lyon.

11. Notamment dans plusieurs collèges et lycées de Perpignan, ville de la branche maternelle de Simon, et à Arbois, village du Jura d'où est issue la branche paternelle. Il semblerait ainsi que l'intérêt pour l'homme facilite l'accès à ses romans. La dimension autobiographique de l'œuvre apparaît comme un appui précieux pour l'enseignant. Voir notamment M. Calle-Gruber, A. Cousin de Ravel et F. Migeot (dir.), Présences de Claude Simon, Mont-de-Laval, L'Atelier du grand Tétras, 2016. Par ailleurs, quelques blogs de professeurs de français proposent des lectures analytiques d'extraits de Simon; p. ex. : [https:// www.samuelhuet.com/linguistique/94-oida/1114-flandres-60.html].

12. Nous reprenons ici des éléments de notre article "Ateliers d'écriture » dans M. Bertrand (dir.), Dictionnaire Claude Simon, t. 1, Champion, 2013.

13. Signalons aussi la proposition de Jean-Luc Vincent qui, dans son ouvrage Comment lire un texte à voix haute?, suggère précisément d'entrer dans le texte par une réflexion sur la meilleure lecture à haute voix possible et par une mise en pratique. Dans un chapitre consacré au "Rythme de la prose ", il prend l'exemple de l'ouverture de La Route des Flandres (jusqu'à "Votre mère m'a écrit ") qu'il considère comme "un défi pour la lecture orale ". Il poursuit : " ce roman est constitué de phrases qui peuvent s'étendre sur plusieurs pages. L'usage extrêmement parcimonieux de la ponctuation, le recours fréquent aux tirets et aux parenthèses, qui viennent interrompre le cours de la phrase, déjà fort sinueux, l'intrusion du style direct non signalé par la ponctuation mais simplement inséré dans le mouvement de la phrase, obligent le lecteur à un examen précis de la syntaxe. La difficulté tient à la nature contradictoire de la contrainte imposée par le texte : faire entendre un flux de paroles ininterrompu et faire entendre le sens. Les pauses respiratoires (lorsqu'elles ne sont pas données par le texte) doivent être compatibles avec le sens et correspondre en même temps à la limite de sa capacité respiratoire, puisqu'il ne s'agit pas de trop respirer un texte lorsqu'il est écrit sans respiration. Le lecteur doit donc faire confiance à l'écriture, qui induit parfois des effets de compréhension qui décalent notre appréhension commune du sens. Comment reproduire l'effet premier que le texte crée chez son lecteur lors de la lecture silencieuse initiale (car il faut bien imaginer que le roman puisse se lire sans que l'on 
c'est d'abord parce que, sur plusieurs points, les manières d'envisager l'écriture - en atelier d'une part, pour Simon d'autre part - sont proches; c'est ensuite parce que l'écriture à partir d'un texte inducteur de Simon amène les élèves non seulement à faire l'expérience d'un rapport à la lecture de cette œuvre différent et stimulant mais aussi à découvrir leurs propres possibilités d'écriture.

Les lecteurs de Claude Simon le savent bien : lire ses romans relève d'une véritable expérience et provoque bien souvent l'envie de faire, de créer, d'écrire. Et c'est parce que Simon a lui aussi fait une expérience similaire qu'il est devenu écrivain. Il affirme dans le Discours de Stockholm : "l'art s'autogénère pour ainsi dire par imitation de lui-même : [...] c'est le désir d'écrire suscité par la fascination de la chose écrite qui fait l'écrivain » (p. 890). Pourquoi ce désir ne ferait-il pas aussi, plus modestement, l' écrivant $^{14}$ " ? Telle est en tous les cas la conviction des animateurs d'ateliers d'écriture créative qui, en présentant aux participants une œuvre littéraire, son auteur, son imaginaire, les traits saillants de son style, espèrent les conduire ensuite, à l'aide d'une consigne, vers l'écriture et le plaisir qu'elle procure. Un autre point commun est à relever : la conception de l'écriture selon Simon et celle des ateliers sont proches en ce sens que pour le romancier, "écrire, c'est, [...] avant tout, faire "15 ", c'est-à-dire travailler le matériau qu'est la langue - le " travail d'écriture ${ }^{16}$ ". Simon revendique son côté « laborieux " et l'emploi d'un « langage d'artisan " (DS, p. 890). Or la connotation artisanale est également présente dans le terme même atelier d'écriture. Comme dans un atelier enfin, Simon valorise le présent de l'acte d'écriture : "C'est seulement en écrivant que quelque chose se produit, dans tous les sens du terme " (préface à Orion aveugle, E I, p. 1181). Il n'en est pas autrement dans un atelier, espace-temps consacré à cet événement qu'est le surgissement des mots, des phrases. Or les livres de Claude Simon font justement partie de ces textes inducteurs privilégiés par les animateurs d'ateliers.

ait besoin de relire chaque page plusieurs fois)?" (Gallimard, 2006, p. 51). Pour lancer cette réflexion, Jean-Luc Vincent suggère de donner le texte écrit puis il fait entendre un enregistrement - le CD est joint à l'ouvrage - qui propose deux lectures : «la première cherche à respecter le sens et la syntaxe sans rendre compte du rythme singulier de l'écriture, la seconde parvient à faire entendre les deux " (ibid.).

14. C'est Dominique Bucheton, qui a axé sa recherche autour de la didactique de l'écriture, qui emploie le terme de "sujet écrivant " dans Refonder l'enseignement de l'écriture, Retz, 2014.

15. C. Simon, "L’inlassable réa/encrage du vécu ", entretiens avec Mireille Calle, dans M. Calle (dir.), Claude Simon. Chemins de la mémoire, Sainte-Foy (Québec)/Grenoble, Le Griffon d'argile/PU de Grenoble, 1993, p. 3.

16. Ibid., p. 5. 
Espace collectif d'échanges, l'atelier, quelle que soit sa visée ou sa philosophie, met en œuvre des pratiques de transmission d'expériences : expériences de lecture, expériences d'écriture. Lorsque les animateurs choisissent de faire écrire leur groupe à partir de textes de Simon, la transmission s'opère le plus souvent autour de l'un des trois éléments suivants : les mots " carrefours de sens " (DS, p. 900), le parti-pris de la description, l'originalité de certains choix énonciatifs. Anne Roche, Andrée Guiguet et Nicole Voltz, enseignantes-chercheures à l'origine de l'introduction des ateliers d'écriture dans le Supérieur en France (à l'université de Lettres d'Aix-en-Provence), auteures de L'Atelier d'écriture, suggèrent ainsi de faire pratiquer la libre association de termes, association "non pas seulement [...] guidée par le sens, mais par les mots et leur matérialité ${ }^{17}$ ». Elles prennent pour exemple un passage des Corps conducteurs, dans lequel ce sont le mot croix, la forme de son référent et une couleur qui motivent la juxtaposition de fragments apparemment sans rapports sémantiques : un personnage casqué tend son index " en direction d'un crucifix que son autre main élève dans le ciel vert» (CC, p. 423) et plus loin : "L’ombre cruciforme de l'avion se déplace rapidement sur une surface pelucheuse ou plutôt crépue, d'un vert uniforme " (p. 424).

Ce sont aussi les techniques de description propres à Simon qui retiennent le plus fréquemment l'attention des animateurs : description de photographies, de cartes postales - dans Histoire notamment -, de tableaux - celui de l'ancêtre dans La Route des Flandres -, autant de supports de la mémoire et de points de départ de reconstitutions hypothétiques et fantasmées du passé grâce à un art subtil du montage. De nombreuses propositions d'écriture ont ainsi pour origine le pouvoir suggestif de l'image iconique. Le réel est également objet de description par excellence, chez Simon. À ceux qui lui demandaient conseil pour écrire, ce dernier répondait : " descendez dans la rue, marchez pendant cent mètres, revenez chez vous et essayez de raconter tout ce que vous avez vu, senti, remémoré ou imaginé pendant ces cent mètres... Vous pouvez avec ça faire un livre énorme ${ }^{18}$... " Les ateliers reprennent cette suggestion, la déclinent. Mais dans tous les cas, elle mène à une authentique réflexion sur l'écriture : comment, dans la linéarité de la prose, rendre la confusion de l'instant, les sensations, les images surgies du

17. A. Roche, A. Guiguet et N. Voltz, L'Atelier d'écriture - Eléments pour la rédaction du texte littéraire, Nathan universités, coll. "Lettres sup », 2000, p. 48.

18. C. Simon, «Attaques et stimuli », dans L. Dällenbach, Claude Simon, Le Seuil, coll. « Les Contemporains ", 1988, p. 180. 
passé? L'un des exercices consiste à enregistrer le réel tel qu'il se présente, dans un inventaire ${ }^{19}$. Simon procède de cette façon lorsqu'il décompose le paysage qui défile devant les yeux d'un observateur, lui-même en mouvement - en l'occurrence dans un train -, rendant ainsi le temps réel sensible :

Par exemple, à partir de la gare de l'Est, noté successivement :

Hangars - Gare de marchandises - Multitudes de voies

Entrepôts - HLM

Poste d'aiguillage

Terrains vagues - Usines

Pavillons - Jardinets (JP, p. 935)

Quant à François Bon, écrivain mais aussi animateur d'ateliers et grand admirateur de l'œuvre de Claude Simon, il propose, dans son ouvrage désormais classique Tous les mots sont adultes, d'explorer les voies de la biographie en s'appuyant, comme dans Les Géorgiques, sur « l'utilisation récurrente d'un il qui va en fait noter ce qu'on sait ou peut imaginer du personnage uniquement en suivant l'ordre d'apparition des figures et images, sans chercher à en produire une mise en ordre chronologique ${ }^{20} »$. Il s'agit d'abord de lire le passage suivant, dans lequel est déroulée l'existence de celui qui n'est pas nommé autrement que par le pronom « il »:

Il a cinquante ans. Il est général en chef de l'artillerie de l'armée d'Italie. Il réside à Milan. Il porte une tunique au col et au plastron brodés de dorures. Il a soixante ans. Il surveille les travaux d'achèvement de la terrasse de son château. Il est frileusement enveloppé d'une vieille houppelande militaire. Il voit des points noirs. Le soir il sera mort. Il a trente ans. Il est capitaine. Il va à l'opéra. Il porte un tricorne, une tunique bleue pincée à la taille et une épée de salon. Sous le Directoire il est ambassadeur à Naples. Il se marie une première fois en 1781 avec une jeune protestante hollandaise. À trente-huit ans il est élu membre de l'Assemblée nationale à la fois dans les départements du Nord et du Tarn. Pendant l'hiver 1807 il dirige le siège de Stralsund en Poméranie suédoise. Il achète un cheval à Friedland. C'est un colosse. Il écrit plaisamment à un ami qu'il a pris trop d'embonpoint pour sa petite taille de cinq pieds neuf pouces. En 1792 il est élu à la Convention. Il écrit à son intendante Batti de veiller à regarnir les haies d'épine blanche. Expulsé de Naples il doit affréter précipitamment un navire génois pour s’enfuir. Il s'associe avec un nommé Garrigou pour l'exploitation des mines de fer de la vallée de l'Aveyron. Il vote la mort du roi. Il est représentant du peuple en mission. [...] De Milan il règle le cérémonial de la visite de l'empereur dans le royaume d'Italie. En pleine Terreur il est élu secrétaire de la Convention et sauve une royaliste qu'il épousera en secondes noces. Un rapport dit de lui qu'il est d'une santé de fer et d'un courage à toute épreuve. Pendant plus d'un an il tient tête en Corse avec moins de douze cents hommes aux insurgés paolistes soutenus par les

19. Voir F. Bon, Tous les mots sont adultes - Méthode pour l'atelier d'écriture, Fayard, 2000, p. 108.

20. Ibid., p. 149. 
escadres de Hood et de Nelson. Il est blessé à la jambe à Farinole. Le navire sur lequel il s'est embarqué à Naples est capturé en mer par un corsaire turc. ( $G$, p. 655-656)

"Répétition saccadée d'une forme épurée, dépouillée, sujet verbe complément, renvoyant immédiatement aux actions objectives du personnage [mais aussi] balises de vie, et reconstructions éminemment subjectives, dans une stricte égalité formelle ${ }^{21}$. "Chaque participant choisit ensuite un personnage qui le touche personnellement mais qui est aussi suffisamment lointain pour qu'il soit entouré d'une certaine aura, et écrit ses actions objectives mais telles qu'elles surgissent dans ses représentations, sans souci de la chronologie : "C'est il ou elle, rien d'autre. Pas de nom. Chaque phrase sera bâtie sur un temps et un lieu, une chose, mais avec chaque fois un saut quant au temps, au lieu, aux choses, jamais deux phrases sur le même continuum ${ }^{22}$."

Dans le cadre d'une part d'un séminaire de master $2 \mathrm{MEEF}$ intitulé "Dire, lire, écrire avec la littérature contemporaine ${ }^{23}$ ", d'autre part d'un module destiné à des enseignants stagiaires de lettres et visant la mise en place d'ateliers d'écriture dans leurs classes de collège ou lycée ${ }^{24}$, nous avons souhaité mettre en œuvre cette même proposition d'atelier d'écriture et avons présenté à chacun des deux groupes le texte en il extrait des Géorgiques. Dans un premier temps, ont été mises en évidence collectivement les spécificités du passage. Pour l'essentiel : l'absence de chronologie au niveau de la diégèse, l'anaphore du $i l$, la présence d'indicateurs spatio-temporels précis, des phrases simples et courtes, l'emploi dominant du présent, l'absence de marques de subjectivité. Chacun a ensuite été invité à écrire pendant vingt minutes son propre texte à partir de la consigne proposée par François Bon puis à lire sa production au groupe. Chaque lecture a fait l'objet d'un retour critique par l'auditoire - chaque auditeur devait prendre des notes pendant la lecture des textes -, retour visant à mettre en avant non seulement l'adéquation de la production aux caractéristiques du texte source mais aussi sa singularité, son originalité. Lors du bilan de cette séance, les stagiaires ont exprimé leur surprise sur plusieurs points : la véritable qualité des textes entendus; la facilité à se mettre à écrire; la capacité à s'approprier le texte de Simon pour écrire un texte personnel; l'émotion ressentie quasiment à chaque lecture à haute voix alors que la consigne d'objectivité avait pourtant bien été respectée. Ils en

21. Ibid.

22. Ibid.

23. ESPE de Paris/université Paris 4, année universitaire 2015-2016.

24. ESPE d'Aix-en-Provence/AMU université, année universitaire 2017-2018. 
ont conclu que la contrainte du texte de départ et de la consigne était étonnamment libératrice mais garantissait aussi selon eux une certaine " tenue formelle » des productions. À la fin de la séance, après relecture de l'extrait des Géorgiques, ils ont surtout découvert par ce retour au texte, c'est ce qui nous intéresse plus particulièrement ici, que c'est la reconstruction d'une vie qui est éminemment subjective et qui provoque l'émotion du lecteur : le choix des moments de vie mis en avant, les parallélismes et échos créés, l'insistance sur certains détails, les effets liés à la juxtaposition de fragments temporels disjoints et non chronologiques. Cette présence de la subjectivité, qui n’avait pas été perçue au départ dans le texte de Simon est alors apparue, après le détour par l'écriture en atelier. L'écriture a donc permis d'affiner la lecture de Simon. Autre effet de cette séance : deux enseignants stagiaires, sur nos conseils, ont lu - et apprécié - respectivement L'Acacia et Le Tramway. On le voit, la situation d'écriture ramène à la lecture; l'atelier d'écriture est aussi le lieu de transmission d'une bibliothèque - simonienne en l'occurrence.

Les stagiaires volontaires ont alors pris à leur tour la position d'animateurs et ont transposé l'atelier dans leurs propres classes, dans la mesure du possible en demi-groupes - de la classe de Cinquième au collège à celle de Seconde générale au lycée. À la suite de la première lecture, les réactions, tous niveaux confondus, ont été vives. Relevons les suivantes, parmi les plus significatives : "on n'y comprend rien "; " on n'a pas le droit d'écrire comme ça " (classe de Cinquième); " c'est bizarre cette façon d'écrire, ça doit être fatigant de lire un roman entier de ce type " (classe de Seconde). Sans s'appesantir, les enseignants stagiaires ont conduit les élèves à identifier le contexte historique et se sont assurés de la compréhension globale de l'extrait. L'atelier s'est ensuite déroulé selon les mêmes modalités que le premier. Les stagiaires ont été surpris cette fois de constater que les élèves, accompagnés, certes, par leur enseignant, avaient repéré les mêmes caractéristiques qu’eux-mêmes dans le texte des Géorgiques. Par ailleurs, les stagiaires ont dans l'ensemble relevé non seulement des productions de qualité ${ }^{25}$ mais aussi des remarques de la part

25. Voici un exemple de production - avec toilettage orthographique - d'un élève de Seconde : "Elle a 63 ans. Elle vit à Clamart avec ses 8 enfants et son mari. Elle porte une tunique kaki et a de nombreuses rides sur son visage. Elle a 9 ans, elle vit paisiblement dans son village. Elle joue à la poupée et aide sa mère à tirer l'eau du puits et à cuisiner le pilaf familial. En 1919, un homme de son village natal vient la voir au couvent des bonnes-sœur et lui propose de se marier avec lui. Elle accepte. En 1900, elle naît avec le siècle dans la maison de ses parents avec l'aide du docteur local. Elle est enveloppée dans un linge blanc et pleure beaucoup. Elle a 15 ans, des soldats vêtus de noir arrivent chez elle et emportent son père. Sa mère pleure beaucoup et de nouvelles personnes viennent s'installer chez eux. Il faut les servir. Elle porte un fichu rouge et des souliers de bois. Elle pense souvent à son père. En 1922, elle 
de l'auditoire dans l'ensemble fondées et objectives. Enfin, la simplicité de la tournure syntaxique attendue dans la production a sans doute libéré les élèves d'habitude les plus récalcitrants à entrer dans l'écriture. Car tous, au dire des stagiaires, ont joué le jeu et la majorité ont même souhaité reconduire la formule de l'écriture en ateliers. Dans plusieurs classes, l'extrait des Géorgiques est devenu un texte de référence, auquel les élèves ont plusieurs fois renvoyé pendant l'année scolaire.

Si Claude Simon n'a pas pour l'instant la place qui lui revient dans les manuels comme dans les classes de lycée, il existe pourtant des voies qui, complémentaires des traditionnelles lectures analytiques, pourraient faciliter l'accès à cette œuvre hors du commun. L'atelier d'écriture créative apparait comme l'une de ces voies. Qu'apporte une telle expérience aux lycéens? François Bon nous livre une réponse : « en récompense, pour les participants, certainement un rapport différent à une œuvre dont la lisibilité ressemble à un parcours en haute montagne, et les joies donc afférentes. Mais aussi un pari : que ces innovations techniques de Claude Simon [...] ouvrent pour chacun de nous des possibilités d'écriture, d'accès à soi-même, infiniment surprenantes et puissantes ${ }^{26}$ ".

débarque à Marseille avec son mari et son premier enfant âgé de 3 mois. L'air y est pur et les mouettes crient. Ils vont prendre un café et demandent l'addition grâce au rudiment d'anglais qu'elle a appris au couvent il y a quelques années de cela. Elle a 16 ans. Sa mère s'est enfuie de sa maison qui ne lui appartient plus depuis 1 an. Elle est morte d'inquiétude pour sa mère et se fait tourmenter par le maître du logis. Elle retrouve sa mère le lendemain, tuée par des soldats. En 1928, elle vient de trouver un logement convenable à Issy-les-Moulineaux dans la banlieue parisienne. Il y a 2 chambres. Une pour elle et son mari et une pour ses 3 enfants qui font beaucoup de bruit la nuit. En 1980, elle meurt d'une pneumonie à l'hôpital du coin. Son fils ainé devient le chef de la famille. Elle a 17 ans. Elle s'enfuit de la maison où elle vit en esclave et arrive à passer la frontière. Elle est recueillie dans un couvent sur l'île de Chypre. Elle porte un uniforme noir et blanc et apprend l'anglais et le latin. En 1935, elle trouve un job de femme de ménage à Clamart et fait les déplacements tous les matins. Elle doit nourrir ses 6 enfants. Elle a 70 ans, tous ses enfants ont fait des études et elle en est fière. Elle est seule maintenant mais sa voisine lui a donné un chat dont elle s'occupe amoureusement. Sa santé commence à décliner. "

26. F. Bon, [http://www.editions-verdier.fr/banquet/n38/atelier.htm]. 